\title{
Physiological signature of late-onset nonallergic asthma of obesity
}

\author{
Anne E. Dixon', Ubong Peters (1) ${ }^{1}$, Ryan Walsh², Nirav Daphtary ${ }^{1}$, \\ Erick S. MacLean ${ }^{1}$, Kevin Hodgdon ${ }^{1}$, David A. Kaminsky ${ }^{1}$ and Jason H.T. Bates ${ }^{1}$
}

Affiliations: ${ }^{1}$ Dept of Medicine, Larner College of Medicine, University of Vermont, Burlington, VT, USA. ${ }^{2}$ Dept of Radiology, Larner College of Medicine, University of Vermont, Burlington, VT, USA.

Correspondence: Jason H.T. Bates, Dept of Medicine, University of Vermont, 149 Beaumont Avenue, HSRF 228, Burlington, Vermont 05405-0075, USA. E-mail: jason.h.batesamed.uvm.edu

\section{ABSTRACT}

Introduction: Obesity can lead to a late-onset nonallergic (LONA) form of asthma for reasons that are not understood. We sought to determine whether this form of asthma is characterised by any unique physiological features.

Methods: Spirometry, body plethysmography, multiple breath nitrogen washout (MBNW) and methacholine challenge were performed in four subject groups: Lean Control $(n=11)$, Lean Asthma $(n=11)$, Obese Control $(n=11)$ and LONA Obese Asthma $(n=10)$. The MBNW data were fitted with a novel computational model that estimates functional residual capacity (FRC), dead space volume $\left(V_{\mathrm{D}}\right)$, the coefficient of variation of regional specific ventilation $\left(\mathrm{C}_{\mathrm{V}, V^{\prime} \mathrm{E}}\right)$ and a measure of structural asymmetry at the level of the acinus $\left(\mathrm{s}_{\mathrm{acin}}\right)$.

Results: Body mass index and waist circumference values were similar in both obese groups, and significantly greater than in lean asthmatic individuals and controls. Forced vital capacity was significantly lower in the LONA Asthma group compared with the other groups $(p<0.001)$. Both asthma groups exhibited similar hyperresponsiveness to methacholine. FRC was reduced in the Obese LONA Asthma group as measured by MBNW, but not in obese controls, whereas FRC was reduced in both obese groups as measured by plethysmography. $\mathrm{V}_{\mathrm{D}}, \mathrm{C}_{\mathrm{V}, V^{\prime} \mathrm{E}}$ and $\mathrm{s}_{\mathrm{acin}}$ were not different between groups.

Conclusions: Chronic lung compression characterises all obese subjects, as reflected by reduced plethysmographic FRC. Obese LONA asthma is characterised by a reduced ability to recruit closed lung units, as seen by reduced MBNW FRC, and an increased tendency for airway closure as seen by a reduced forced vital capacity.

@ERSpublications

Obesity increases the risk of developing a late-onset nonallergic form of asthma. This form of obese asthma may be related to an increased tendency towards airway closure and inability to open the lungs with a deep breath. https://bit.ly/3eRdXv6

Cite this article as: Dixon AE, Peters $\mathrm{U}$, Walsh $\mathrm{R}$, et al. Physiological signature of late-onset nonallergic asthma of obesity. ERJ Open Res 2020; 6: 00049-2020 [https://doi.org/10.1183/ 23120541.00049-2020]. 


\section{Introduction}

Asthma, like many chronic diseases, is adversely affected by obesity. In some cases, this manifests as a modulation of the asthma phenotype typically seen in lean populations, resulting in more severe symptoms and frequent exacerbations [1]. Obesity also causes de novo asthma, but with a disease phenotype that differs from that seen in lean individuals. While disease in lean individuals often has an allergic basis and first presents in childhood, the obesity-specific form of asthma often presents later in life and is not associated with allergy or type-2 inflammation [2]. Furthermore, airway reactivity in this late-onset nonallergic (LONA) form of obese asthma improves dramatically with weight loss, suggesting that its pathophysiology may be quite different from that of the conventional form of asthma that affects lean individuals [3]. Obese patients also tend to have worse asthma control, and their disease does not respond as well to many standard therapies [4]. Elucidating the mechanistic basis of LONA obese asthma is important for the development of appropriate therapies to treat this patient population.

It is easy to imagine that LONA obese asthma might have something to do with the chronic reductions in lung volume caused by mass loading of the chest wall. It has been shown, for example, in both humans [5] and animal models $[6,7]$, that airways' responsiveness to methacholine challenge is greatly enhanced when volume descends below normal functional residual capacity (FRC). Correspondingly, reduced lung volumes in obese individuals might thus lead them also to exhibit asthma-like hyperresponsiveness. This notion is supported by the finding that obese asthma incidence and prevalence correlates with body mass index (BMI) $[8,9]$. Nevertheless, even with BMIs of $45 \mathrm{~kg} \cdot \mathrm{m}^{-2}$ and above, most obese individuals do not develop LONA asthma [9]. This raises the question as to what is special about those who do.

There are a number of physiological mechanisms that could potentially lead to excessive impairment of lung function at low lung volumes in only some obese subjects. For example, individuals with LONA obese asthma might have airways that are simply more prone to collapse than average [10, 11], or they might have a lipid status that is altered enough to impair surfactant function [12], or they might have elevated levels of systemic inflammation that lead to remodelled airways [13] that are at risk for becoming occluded. Such theories are still speculative and thus remain in strong need of investigation. To gain further insight into this question we therefore assessed the physiological characteristics of individuals with LONA obese asthma relative to those of obese individuals without asthma. Since LONA obese asthma appears to be a disease of altered lung mechanics, we focused on the measurement of characteristics that reflect both overall lung function as well as its degree of regional heterogeneity. We also measured these characteristics in both lean control and lean asthmatic subjects in order to provide a basis of comparison against the physiological factors that predispose to conventional allergic asthma.

\section{Methods}

\section{Human subjects}

The study protocol was approved by the University of Vermont Institutional Review Board. Informed consent was obtained from all participants. Inclusion criteria were as follows: LONA asthma - physician diagnosis of asthma at age $\geqslant 18$ years, $\mathrm{BMI} \geqslant 35 \mathrm{~kg} \cdot \mathrm{m}^{-2}$, ages $35-55$ years, serum $\mathrm{IgE}<100 \mathrm{IU} \cdot \mathrm{mL}^{-1}$; obese control - no history of asthma, $\mathrm{BMI} \geqslant 35 \mathrm{~kg} \cdot \mathrm{m}^{-2}$, ages $35-55$ years, serum $\mathrm{IgE}<100 \mathrm{IU} \cdot \mathrm{mL}^{-1}$; lean control no history of asthma, BMI $18.5-24.9 \mathrm{~kg} \cdot \mathrm{m}^{-2}$, ages $35-55$ years, serum $\mathrm{IgE}<100 \mathrm{IU} \cdot \mathrm{mL}^{-1}$; lean asthma physician diagnosis of asthma at age $<18$ years, BMI $18.5-24.9 \mathrm{~kg} \cdot \mathrm{m}^{-2}$, ages $18-55$ years, serum $\mathrm{IgE} \geqslant 100 \mathrm{IU} \cdot \mathrm{mL}^{-1}$. We did not perform testing for aeroallergens, but all allergic asthmatic patients had elevated IgE and reported a history of seasonal allergies and/or allergies worsening their asthma. The original protocol included only lean allergic asthmatic subjects ages 35-55 years, but because of difficulty recruiting such participants, the eligibility criteria were modified to include younger participants. Participants with forced expiratory volume in $1 \mathrm{~s}\left(\mathrm{FEV}_{1}\right)<60 \%$ predicted, other forms of lung disease, exacerbation within the last 8 weeks, pregnancy and $\geqslant 20$ pack-years smoking history were excluded.

\section{Questionnaires and lung function measurement}

Asthma control was assessed using the Juniper Asthma Control Questionnaire [14]. Spirometry, lung volumes and single breath nitrogen washouts were measured (Platinum Elite ${ }^{\mathrm{TM}}$ body plethysmograph; MGC Diagnostics, Saint Paul, MN, USA) according to American Thoracic Society (ATS) and European Respiratory Society (ERS) guidelines [15-17]. Methacholine challenge testing, assessed by spirometry, was performed according to the 1999 ATS guidelines using the two-minute tidal breathing protocol [18], administering a maximum concentration of methacholine of up to $16 \mathrm{mg} \cdot \mathrm{mL}^{-1}$ for participants with asthma, and up to $64 \mathrm{mg} \cdot \mathrm{mL}^{-1}$ for controls.

\section{Multiple breath nitrogen washout}

Multiple breath nitrogen washout (MBNW) was measured using Easy One Pro (ndd Medical Technologies, Andover, MA, USA) according to ATS/ERS guidelines [16]. While subjects sat comfortably 
in a chair attempting to breathe pure oxygen in a deep and regular manner, the nitrogen fraction and airflow $\left(V^{\prime} \mathrm{E}\right)$ at the mouth were monitored continuously at $200 \mathrm{~Hz}$ until nitrogen fraction at the end of Phase III during expiration had fallen to $<1 / 40$ of its baseline value. Each subject performed several washout manoeuvres with a rest between each. All subjects produced at least one manoeuvre that was judged to be technically acceptable in terms of exhibiting a monotonic decrease in the breath-by-breath expired nitrogen fraction over the entire course of the washout. Most subjects produced three acceptable manoeuvres.

The nitrogen fraction and flow signals recorded throughout each manoeuvre were fitted with a novel computational model that we recently introduced [19]. This model assumes the lung to be comprised of a large number of parallel units that differ only in their respective tidal volumes and the changing relative contributions they each make to flow as expiration proceeds. The model provides estimates of four quantities of physiological interest - FRC, the volume of the dead space $\left(V_{D}\right)$, the coefficient of variation of specific ventilation throughout the entire lung $\left(\mathrm{C}_{\mathrm{V}, V_{\mathrm{E}}^{\prime}}\right)$ and a parameter that reflects local structural asymmetry in the airway tree at the level of the acinus $\left(\mathrm{s}_{\text {acin }}\right)$. The parameters obtained from the model fit with the smallest mean squared residual between the measured and predicted nitrogen fraction for each subject were retained for analysis [19].

Compared to the conventional approach of determining the slope $\left(\mathrm{s}_{\mathrm{cond}}\right)$ and intercept $\left(\mathrm{s}_{\text {acin }}\right)$ of the normalised Phase III slope versus cumulative expired volume [20], the model-fitting approach has the advantage of not requiring identification of the transition point between Phase II and Phase III of the alveolar plateau in nitrogen fraction. It also does not require that subjects breathe in a deep and uniform manner throughout the manoeuvre, something that a number of our subjects struggled to do.

\section{Statistics}

Statistical analysis was performed using STATA 16.0 (Statacorp, College Station, TX, USA). Differences across groups were tested by ANOVA and Kruskal-Wallis test. Group pairs were compared by t-test with Bonferroni correction. Statistical significance was interpreted as $\mathrm{p}<0.05$.

\section{Results}

Table 1 lists the demographics of the four study groups, which were Lean Control ( $\mathrm{n}=11)$, Lean Asthma $(n=11)$, Obese Control $(n=11)$ and Obese LONA Asthma $(n=10)$. The groups were well matched in terms of age with the exception of the Lean Asthma subjects who were roughly half the age of the other groups (related to difficulty identifying participants aged 35-55 years with early-onset allergic asthma who were lean). The two obese groups were similar in body habitus, although the obese controls were slightly more obese than the obese LONA asthmatic individuals and had somewhat greater waist circumference (table 1). The Obese Asthma group had worse asthma control and more urgent care visits than the Lean Asthma group (table 2). Forced vital capacity (FVC) in the Obese LONA Asthma group was significantly lower than the other three groups (table 3). Total lung capacity (TLC) was numerically lower in the Obese Asthma group. FRC, measured by plethysmography, was significantly lower in both obese groups compared with both lean groups.

Figure 1 shows the mean components of TLC measured by plethysmography in each of the four groups expressed as fractions of $100 \%$ predicted TLC. This figure illustrates how obesity and asthma, both alone and in combination, potentially alter lung volume. In particular, the hatched areas in figure 1 represent the predicted volumes of consolidated lung responsible for preventing each group from reaching $100 \%$ TLC. What this figure suggests is that although FRC is depressed in both obese groups, the obese controls are

\begin{tabular}{|c|c|c|c|c|c|}
\hline & \multicolumn{2}{|c|}{ Lean } & \multicolumn{2}{|c|}{ Obese } & \multirow[t]{2}{*}{ p-value } \\
\hline & Control $(n=11)$ & Asthma (n=11) & Control (n=11) & Asthma $(n=10)$ & \\
\hline Sex $\mathbf{n}$ & $8 F / 3 M$ & $9 F / 2 M$ & $8 F / 3 M$ & $7 F / 3 M$ & 0.92 \\
\hline Age years & $41.7 \pm 6.1$ & $23.1 \pm 1.6$ & $44.6 \pm 4.1$ & $45.2 \pm 6.9$ & $<0.01$ \\
\hline $\mathrm{BMI} \mathbf{k g} \cdot \mathrm{m}^{-2}$ & $22.5 \pm 1.5$ & $23.1 \pm 1.7$ & $44.8 \pm 4.1$ & $42.4 \pm 9.1$ & $<0.01$ \\
\hline Smoking pack-years & $0.14 \pm 0.31$ & 0 & $2.05 \pm 2.31$ & $2.14 \pm 4.75$ & 0.12 \\
\hline Waist circumference $\mathrm{cm}$ & $86.6 \pm 9.8$ & $82.6 \pm 8.1$ & $139.0 \pm 9.5$ & $117.4 \pm 23.7$ & $<0.01$ \\
\hline Age of asthma onset years & - & $4(1,16)$ & - & $27(18,50)$ & \\
\hline
\end{tabular}

Data are presented as mean \pm SD or median (range), unless otherwise stated. F: female; M: male; BMI: body mass index. 


\begin{tabular}{|c|c|c|c|}
\hline & Lean & Obese & p-value \\
\hline \multicolumn{4}{|l|}{ Medication use } \\
\hline ICS-LABA-LAMA & 0 & 1 & 0.31 \\
\hline ICS-LABA & 2 & 2 & 1 \\
\hline ICS & 1 & 2 & 0.53 \\
\hline LTRA & 2 & 0 & 0.14 \\
\hline No controller therapy & 6 & 7 & 0.86 \\
\hline \multicolumn{4}{|l|}{ Asthma control score } \\
\hline$A C Q$ score & $0.84 \pm 0.71$ & $1.63 \pm 0.63$ & $0.01^{\Re}$ \\
\hline \multicolumn{4}{|c|}{ Urgent asthma care in the prior 12 months } \\
\hline ED visit & 0 & 1 & 0.31 \\
\hline Urgent care (non-ED) visit & 0 & 4 & 0.03 \\
\hline Systemic corticosteroids & 1 & 2 & 0.53 \\
\hline \multicolumn{4}{|c|}{$\begin{array}{l}\text { Data are presented as number of participants or mean } \pm \text { SD, unless otherwise stated. ICS: inhaled } \\
\text { corticosteroid; LABA: long-acting beta-agonist; LAMA: long-acting muscarinic antagonist; LTRA: } \\
\text { leukotriene receptor antagonist; ACQ: Asthma Control Questionnaire; ED: emergency department. } \\
\text { p-values were calculated using the Chi-squared test, except ", which was calculated using the t-test. } \\
\# \text { : higher score is indicative of worse asthma control. }\end{array}$} \\
\hline
\end{tabular}

still able to approach a normal TLC with a deep inspiration. In contrast, in the Obese LONA Asthma group TLC is somewhat reduced compared to the obese controls, suggesting less recruitable lung tissue in the Obese Asthma group.

Model analysis of MBNW data showed that FRC was significantly lower in the Obese LONA Asthma group compared to the other three groups (figure 2, top panel). In contrast, there were no differences between the groups in $\mathrm{V}_{\mathrm{D}}$ (figure 2, bottom panel) or in the two model parameters reflecting ventilation heterogeneity $\left(\mathrm{C}_{\mathrm{V}, V_{\mathrm{E}}^{\prime}}\right.$ and $\mathrm{s}_{\mathrm{acin}}$ figure 3$)$. The model fit the data from each group equally well, as there were no differences between the four groups in the root mean squared residual (RMSR) in nitrogen fraction between the data and the fits. The mean (SD) values of RMSR from the four groups were Lean Control $=0.00984$ (0.001750), Lean Asthma $=0.00951$ (0.00165), Obese Control $=0.01007$ (0.00246), Obese LONA Asthma $=0.00901(0.00217)$. The tidal volumes during the washout tests were essentially identical in the Lean Control, Lean Asthma and Obese Control groups at $0.85 \pm 0.26,0.86 \pm 0.61$ and $0.82 \pm 0.22 \mathrm{~L}$,

TABLE 3 Lung function data

\begin{tabular}{|c|c|c|c|c|c|}
\hline & \multicolumn{2}{|c|}{ Lean } & \multicolumn{2}{|c|}{ Obese } & \multirow[t]{2}{*}{ p-value ${ }^{\#}$} \\
\hline & Control & Asthma & Control & Asthma & \\
\hline $\mathrm{FEV}_{1} \%$ pred & $99.1 \pm 9.2^{*}$ & $89.2 \pm 16.6$ & $99.0 \pm 12.3$ & $83.0 \pm 13.6$ & 0.02 \\
\hline FVC \% pred & $104.3 \pm 5.4^{*}$ & $98.5 \pm 14.7 *$ & $96.9 \pm 11.9 *$ & $80.9 \pm 9.8$ & $<0.001$ \\
\hline FEV $_{1} /$ FVC $\%$ & $77.0 \pm 5.7$ & $76.7 \pm 8.5$ & $82.0 \pm 6.9$ & $83.1 \pm 4.2$ & 0.051 \\
\hline ERV \% pred & $62.5 \pm 39.8$ & $69.1 \pm 29.7$ & $44.3 \pm 35.4$ & $51.4 \pm 46.5$ & 0.53 \\
\hline TLC \% pred & $103.4 \pm 8.72 *$ & $98.2 \pm 14.0$ & $96.1 \pm 12.8$ & $87.4 \pm 8.9$ & 0.02 \\
\hline RV \% pred & $132.4 \pm 39.4^{*}$ & $129.1 \pm 36.5^{*}$ & $99.5 \pm 30.4$ & $82.7 \pm 36.9$ & $<0.01$ \\
\hline RV/TLC \% pred & $128.3 \pm 30.2$ & $136.3 \pm 42.5^{*}$ & $102.5 \pm 26.8$ & $93.9 \pm 35.5$ & 0.02 \\
\hline FRC \% pred & $96.0 \pm 22.8^{+}$ & $98.6 \pm 10.0^{+}$ & $76.2 \pm 11.9$ & $73.9 \pm 16.6$ & $<0.001$ \\
\hline$s G_{\text {aw }} \%$ pred & $\begin{array}{c}119 \pm 92.0 \\
50(222-64)^{\pi}\end{array}$ & $\begin{array}{c}127.9 \pm 72.6 \\
28(01-128)\end{array}$ & $\begin{array}{l}107.8 \pm 78.1 \\
64(64-64)^{9}\end{array}$ & $156.6 \pm 92.9$ & 0.59 \\
\hline
\end{tabular}

Data are presented as mean \pm SD or median (range), unless otherwise stated. Predicted values for spirometry are based on NHANES III [21], and those for lung volumes are based on GoLDMAN and BECKLAKE [22]. $\mathrm{FEV}_{1}$ : forced expiratory volume in $1 \mathrm{~s} ; \%$ pred: \% predicted; FVC: forced vital capacity; ERV: expiratory reserve volume; TLC: total lung capacity; RV: residual volume; FRC: functional residual capacity; $s G_{a w}$ : specific airway conductance; $\mathrm{PC}_{20}$ : provocative concentration causing a $20 \%$ fall in $\mathrm{FEV}_{1} .{ }^{\#}$ : $\mathrm{p}$-value shown for one-way analysis of variance for normally distributed variables, and Kruskal-Wallis test for non-parametric variables; *: $p<0.05$ compared with Obese Asthma group, post hoc comparison with Bonferroni correction; ": $p<0.05$ compared with asthma groups, post hoc comparison with Bonferroni correction; ${ }^{+}: p<0.05$ compared with obese groups, post hoc comparison with Bonferroni correction. 
FIGURE 1 Mean values for total lung capacity (TLC), functional residual capacity (FRC) and residual volume (RV) in each of the four study groups, expressed as fractions of $100 \%$ predicted TLC for each group as measured by plethysmography. The Lean Control group slightly exceeded 100\% predicted TLC. Mean TLC in each of the remaining groups was $<100 \%$ predicted by amounts that are potentially attributable to volumes of atelectatic (non-recruitable) lung (grey).

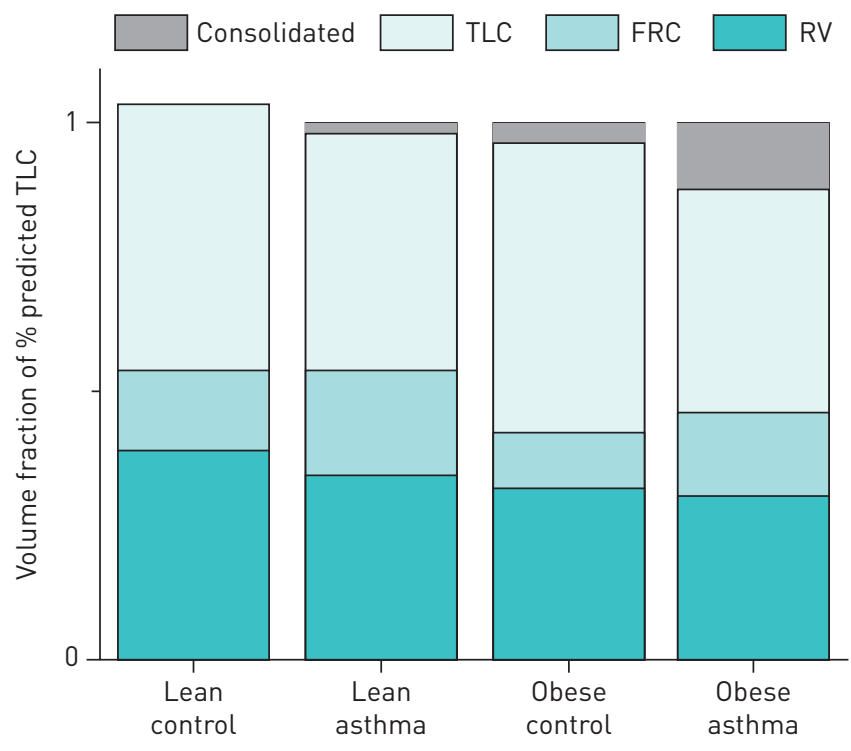

respectively (mean $\pm \mathrm{sD})$, but were significantly less $(\mathrm{p}=0.02$, ANOVA) in the LONA Asthma group at $0.62 \pm 0.26 \mathrm{~L}$. None of the correlations between tidal volume and $\mathrm{V}_{\mathrm{D}}$ for any of the groups was significant $(\mathrm{p}>0.05)$.

\section{Discussion}

Although obesity is clearly linked to asthma [23], a critical unanswered question is why LONA asthma develops in only some obese individuals while the remainder are spared. In the present study, we have attempted to shed light on this puzzling situation by studying how key lung volumes are differentially affected by obesity and asthma, both individually and in combination, since it is easy to imagine that chronic compression of the lungs by the obese chest wall somehow plays a pathogenic role. We studied lean individuals with allergic asthma (as a common form of asthma in lean individuals), but studied late-onset nonallergic asthma in obese participants: we have identified LONA asthma as having distinct features compared with obese allergic asthma, the latter likely representing a complex phenotype of asthma complicated by obesity, rather than asthma consequent to obesity $[3,11,24]$. However, this cannot be the whole story because mass loading of the lungs by the chest wall is common to all obese subjects, giving them a kind of restrictive physiology $[25,26]$. Indeed, while FRC measured plethysmographically was substantially reduced in obese control versus lean subjects, it was very similar in both obese groups (table 3), suggesting that a reduced operating lung volume is a feature of obesity in general. In fact, if anything, we might have expected lung compression to be greater in the Obese Control group because they had slightly greater BMI compared to the Obese LONA Asthma group. Waist circumference, a marker of
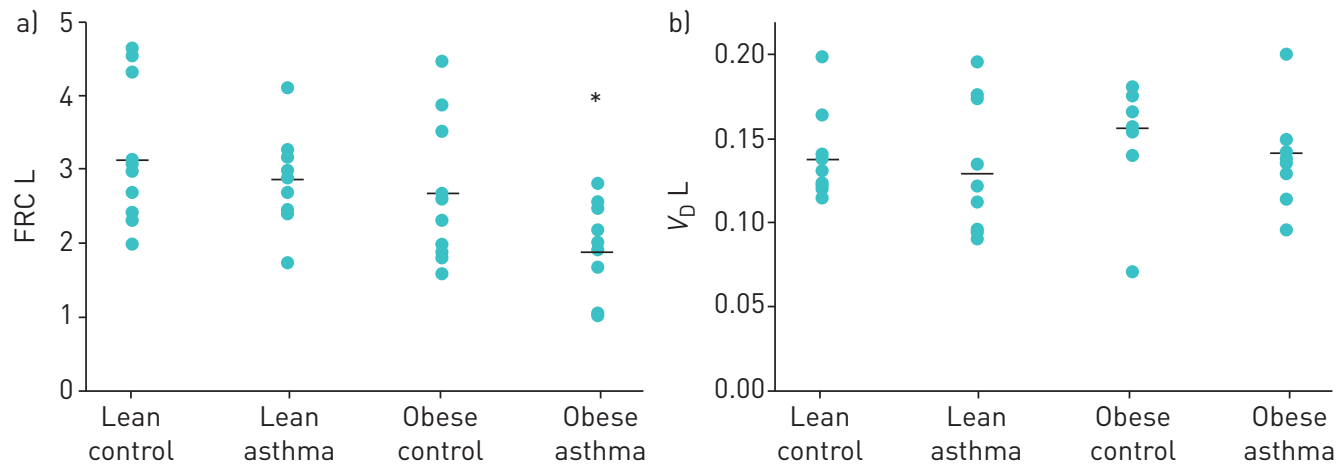

FIGURE 2 Closed symbols show the parameters derived from multiple breath nitrogen washout pertaining to lung size (a: functional residual capacity (FRC) and b: dead space volume $\left(V_{D}\right)$ for each of the four study groups (means shown as horizontal bars). FRC from nitrogen washout was significantly lower in the late-onset nonallergic (LONA) obese asthma group compared to FRC from nitrogen washout in the other groups (*: ANOVA and paired t-test, $p<0.05)$. 
a) 60

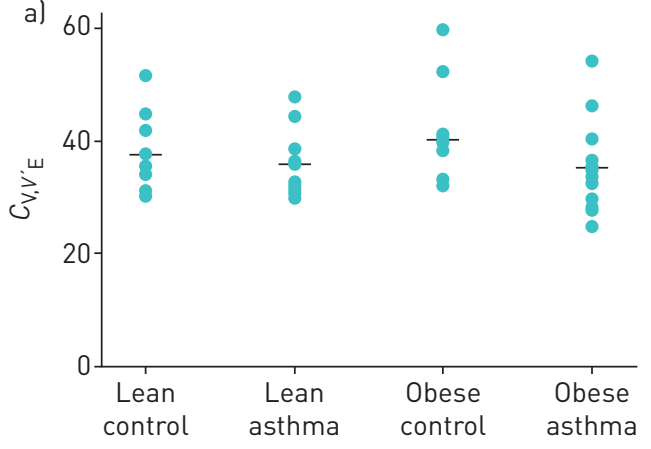

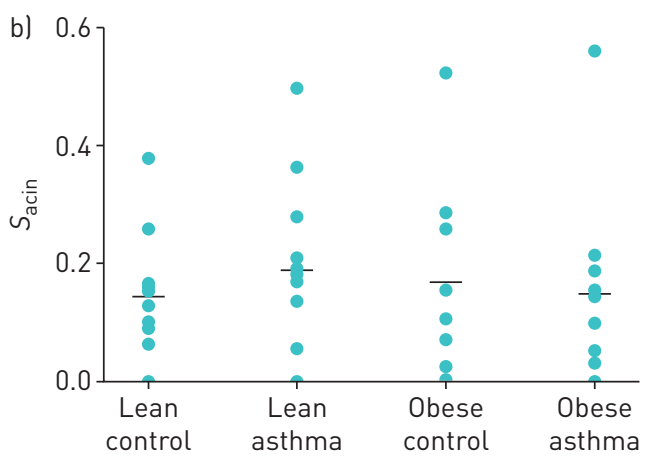

FIGURE 3 Parameters derived from multiple breath nitrogen washout pertaining to lung heterogeneity la: the coefficient of variation of regional specific ventilation $\mathrm{C}_{V_{1} V_{E}^{\prime}}$ and $\mathrm{b}$ : a measure of structural asymmetry at the level of the acinus $\left(s_{a c i n}\right)$ ) for each of the four study groups (means shown as horizontal bars). There were no significant differences between groups for either parameter.

abdominal fat content, was also somewhat greater in the Obese Control group (table 1). Nevertheless, both obese groups had virtually identical $\mathrm{FEV}_{1} / \mathrm{FVC}$ (table 3 ).

One feature that significantly differed in the LONA Obese Asthma group, compared to all other groups, was reduced FVC. Few other studies have compared spirometry in adults with obese asthma and obese controls, though both our own group and others have previously reported a reduction in FVC in obese asthmatic individuals compared to obese controls $[3,27]$. In contrast, prior studies in children report similar FVC in obese children with asthma and obese controls [28], which might reflect effects of obesity on lung growth in children [29]. We recruited adults with late-onset asthma, and so these participants were likely a distinct phenotype compared with obese children. Other lung volumes and flow rates also tended to be lower in obese asthma compared with other groups (such as $\mathrm{FEV}_{1}$, TLC and residual volume), but only FVC was significantly reduced. Insofar as reduction in FVC reflects airway closure during forced exhalation, the fact that FVC appeared to be a particular characteristic of obese asthma likely reflects instability and derecruitment of the airways during forced exhalation. We cannot be sure at which level in the airway tree this instability manifests, but it seems likely to involve the small airways since dysfunction at this level has been shown to be a key feature of asthma in general [30].

The other observation that supports derecruitment of the airways is our MBNW data. Although plethysmographic FRC was decreased in both obese groups (table 3), suggestive of the compressive effects of adipose tissue around the chest wall, as has been reported by others [31], FRC measured by nitrogen washout was reduced only in the Obese LONA Asthma group (figure 2). At the same time, the physical sizes of the lungs in the two obese groups appear to have been similar because $V_{D}$ was not different between the groups (figure 2). (It is possible that a difference in $V_{D}$ between the LONA Asthma group and the other three groups was masked by the fact that tidal volume was about $25 \%$ lower during the washout manoeuvres in the LONA Asthma group, although the lack of a significant correlation between $\mathrm{V}_{\mathrm{D}}$ and tidal volume in all groups makes this less likely.) These apparently contradictory findings can be explained on the basis of differences in the two FRC measurement methods. FRC is measured plethysmographically by having subjects perform small-amplitude panting manoeuvres that involve minimal changes in lung volume and thus reflect quasi-static lung volume at FRC. In contrast, nitrogen washout measurements require subjects to take large breaths above FRC. If subjects with obese LONA asthma recruit less lung volume during these breaths compared to obese controls, then they will experience less dilution of alveolar gas by the inspired oxygen and will thus register correspondingly reduced FRC values. Thus, the apparent anomaly between the plethysmographic and nitrogen washout measurements of FRC supports the notion, articulated above, that obese LONA asthmatic patients have difficulty fully recruiting their lungs on deep inspiration, while the obese non-asthmatic subjects experience no such difficulty.

Interestingly, there were no differences in parameters reflecting ventilation heterogeneity between asthmatic and non-asthmatic groups (figure 3). This might, however, be explained by the fact that the lean asthmatic subjects had disease that was either mild or well controlled. Indeed, we have previously found similar levels of heterogeneity in patients with mild asthma versus controls during similar levels of bronchoconstriction [32]. Also, a normal degree of heterogeneity in the obese LONA subjects would be expected if their disease was due to increased derecruitment because the remaining open lung would still be relatively normal. Mild disease would also explain why there are no obvious differences between the 
plethysmographic and washout values of FRC in figure 2; gas trapping in severe obstructive lung disease can cause plethysmographic FRC values to be higher than washout values [17].

There are several possible explanations for why obese subjects with LONA asthma could suffer from increased derecruitment of lung units that is difficult to reverse. One possibility is increased smooth muscle tone [33, 34]; changes in airway smooth muscle structure and function have been reported in obesity [33], and decreased levels of endogenous nitric oxide could contribute to increased airway smooth muscle tone. Indeed, Holguin et al. [35] showed that supplementation with L-citrulline to specifically increase nitric oxide in obese patients with asthma particularly improved pre-bronchodilator FVC. Another possibility is the presence of metabolic dysfunction that might affect airway remodelling [13] to cause increases in wall thickness and secretion volume, both of which are potent mechanisms for enhanced airway closure [36]. However, while visceral adipose inflammation is increased in obese LONA asthma compared to obese controls [2,37], this inflammation does not seem to manifest in the airways [2]. Yet another possible mechanism for increased lung derecruitment is decreased surfactant function, which could potentially occur in obese LONA asthma as a result of alterations in fatty acids due to metabolic dysfunction [12]. Increased surface tension in the lungs due to surfactant dysfunction is an extremely potent mechanism for instability and collapse of alveoli and small airways in the acutely injured lung $[38,39]$, so even modest alterations to the amount and/or composition of surfactant could have clinically significant effects in obesity.

Finally, although our findings concerning the distinguishing characteristic of obese LONA asthma suggest possibilities for their mechanistic bases, these must be viewed in light of the study's limitations. Principal among these is that our four study groups were small, containing 10-11 subjects each. While these numbers might be sufficient to reveal statistically significant differences in certain physiological parameters, they hardly constitute representative samplings of the heterogeneous populations from which they were drawn. Lean allergic asthmatic individuals were younger than the other groups, and so this might also contribute to differences, but as their data were similar to those of lean controls, age likely did not have a large effect. The LONA obese participants had more severe asthma than the lean participants with asthma did, and so this might also have contributed to differences between groups. We did not study allergic obese asthmatics in the present study as this was not central to our central question of what distinguishes LONA obese asthmatics from obese non-asthmatic subjects, but this is certainly something that bears future investigation. Also, although we used a number of different investigative methods spirometry, plethysmography and MBNW - to study our subjects, these methods reveal phenotype only. Accordingly, any mechanistic conclusions we have drawn remain inferential, particularly as the form of hyperresponsiveness we focus on here is peculiar to obesity and clearly quite different from that occurring in conventional asthma. In addition, our method of analysis of MBNW data relies on a model of ventilation distribution in the lung that, although providing quite good fits to the data, is inevitably an imperfect representation of the real organ, so the parameter values we obtained by this method likely reflect these model imperfections to a degree that is difficult to quantify. Strengths of the study include the careful phenotyping of the groups, and age and body habitus matching of the obese groups. Even so, there remain other investigational techniques that could have been brought to bear on the questions we address here. Important among these is the measurement of respiratory system impedance by the technique of oscillometry, which has proven useful in discriminating between control and asthmatic subjects [11, 30, 40].

In summary, our data suggest that individuals with obese LONA asthma have abnormalities of airway function that distinguish them from obese controls as well from lean subjects either with or without asthma. Specifically, obese individuals with LONA asthma appear to have exaggerated derecruitment with forced exhalation, and an impaired capacity to reopen derecruited regions of the lung when they take a deep breath. The mechanisms behind this phenotype remain unclear but could involve processes that remodel the airway wall and increase secretions, and increase smooth muscle tone, and/or metabolic processes that decrease the ability of surfactant to lower surface tension in the lung. Therapies that target derecruitment may have an important role in the treatment of late-onset asthma in obese patients.

Conflict of interest: A.E. Dixon reports that MitoQ supplied the study drug for a clinical trial outside the submitted work. U. Peters has nothing to disclose. R. Walsh has nothing to disclose. N. Daphtary has nothing to disclose. E.S. MacLean has nothing to disclose. K. Hodgdon has nothing to disclose. D.A. Kaminsky reports personal fees from MGC Diagnostics, Inc., for participating as faculty on a course outside the submitted work. J.H.T. Bates reports grant R01 HL130847 from the NIH-NHLBI during the conduct of the study. He is a consultant for an minor shareholder in Oscillavent, LLC (Iowa), and a consultant for Healthy Design, LLC (Vermont), outside the submitted work. In addition, he has patents (Device and method for lung measurement, US patent application US 20160007882 A1; Variable ventilation as a diagnostic tool for assessing lung mechanical function, PCT Application WO2015127377 A1) pending.

Support statement: This study was supported by NIH grant R01 HL-130847. Funding information for this article has been deposited with the Crossref Funder Registry. 


\section{References}

1 Holguin F, Bleecker ER, Busse WW, et al. Obesity and asthma: an association modified by age of asthma onset. J Allergy Clin Immunol 2011; 127: 1486-1493 e1482.

2 Sideleva O, Suratt BT, Black KE, et al. Obesity and asthma: an inflammatory disease of adipose tissue not the airway. Am J Respir Crit Care Med 2012; 186: 598-605.

3 Dixon AE, Pratley RE, Forgione PM, et al. Effects of obesity and bariatric surgery on airway hyperresponsiveness, asthma control, and inflammation. J Allergy Clin Immunol 2011; 128: 508-515.e501-502.

4 Peters U, Dixon AE, Forno E. Obesity and asthma. J Allergy Clin Immunol 2018; 141: 1169-1179.

5 Ding DJ, Martin JG, Macklem PT. Effects of lung volume on maximal methacholine-induced bronchoconstriction in normal humans. J Appl Physiol 1987; 62: 1324-1330.

6 Bates JH, Lauzon AM, Dechman GS, et al. Temporal dynamics of pulmonary response to intravenous histamine in dogs: effects of dose and lung volume. J Appl Physiol 1994; 76: 616-626.

7 Cojocaru A, Irvin CG, Haverkamp HC, et al. Computational assessment of airway wall stiffness in vivo in allergically inflamed mouse models of asthma. J Appl Physiol 2008; 104: 1601-1610.

8 Beuther DA, Sutherland ER. Overweight, obesity, and incident asthma: a meta-analysis of prospective epidemiologic studies. Am J Respir Crit Care Med 2007; 175: 661-666.

9 The LABS Writing Group, for the LABS Consortium, Belle SH, Chapman W, et al. Relationship of body mass index with demographic and clinical characteristics in the Longitudinal Assessment of Bariatric Surgery (LABS) Surg Obes Relat Dis 2008; 4: 474-480.

10 Bates JH, Dixon AE. Potential role of the airway wall in the asthma of obesity. J Appl Physiol 2015; 118: 36-41.

11 Al-Alwan A, Bates JH, Chapman DG, et al. The nonallergic asthma of obesity. A matter of distal lung compliance. Am J Respir Crit Care Med 2014; 189: 1494-1502.

12 McGillick EV, Lock MC, Orgeig S, et al. Maternal obesity mediated predisposition to respiratory complications at birth and in later life: understanding the implications of the obesogenic intrauterine environment. Paediatr Respir Rev 2017; 21: 11-18.

13 Barton JH, Ireland A, Fitzpatrick M, et al. Adiposity influences airway wall thickness and the asthma phenotype of HIV-associated obstructive lung disease: a cross-sectional study. BMC Pulm Med 2016; 16: 111.

14 Juniper EF, O'Byrne PM, Guyatt GH, et al. Development and validation of a questionnaire to measure asthma control. Eur Respir J 1999; 14: 902-907.

15 Miller MR, Hankinson J, Brusasco V, et al. Standardisation of spirometry. Eur Respir J 2005; 26: 319-338.

16 Robinson PD, Latzin P, Verbanck S, et al. Consensus statement for inert gas washout measurement using multiple- and single-breath tests. Eur Respir J 2013; 41: 507-522.

17 Wanger J, Clausen JL, Coates A, et al. Standardisation of the measurement of lung volumes. Eur Respir J 2005; 26 511-522.

18 Crapo RO, Casaburi R, Coates AL, et al. Guidelines for methacholine and exercise challenge testing - 1999. This official statement of the American Thoracic Society was adopted by the ATS Board of Directors, July 1999. Am J Respir Crit Care Med 2000; 161: 309-329.

19 Bates JHT, Peters U. A model-based approach to interpreting multibreath nitrogen washout data. J Appl Physiol 2018; 124: 1155-1163.

20 Verbanck S, Thompson BR, Schuermans D, et al. Ventilation heterogeneity in the acinar and conductive zones of the normal ageing lung. Thorax 2012; 67: 789-795.

21 Hankinson JL, Odencrantz JR, Fedan KB. Spirometric reference values from a sample of the general U.S population. Am J Respir Crit Care Med 1999; 159: 179-187.

22 Goldman HI, Becklake MR. Respiratory function tests; normal values at median altitudes and the prediction of normal results. Am Rev Tuberc 1959; 79: 457-467.

23 Dixon AE, Poynter ME. Mechanisms of asthma in obesity. Pleiotropic aspects of obesity produce distinct asthma phenotypes. Am J Respir Cell Mol Biol 2016; 54: 601-608.

24 Chapman DG, Irvin CG, Kaminsky DA, et al. Influence of distinct asthma phenotypes on lung function following weight loss in the obese. Respirology 2014; 19: 1170-1177.

25 Jones RL, Nzekwu MM. The effects of body mass index on lung volumes. Chest 2006; 130: 827-833.

26 Littleton SW, Tulaimat A. The effects of obesity on lung volumes and oxygenation. Respir Med 2017; 124: 15-20.

27 Nicolacakis K, Skowronski ME, Coreno AJ, et al. Observations on the physiological interactions between obesity and asthma. J Appl Physiol 2008; 105: 1533-1541.

28 Rastogi D, Fraser S, Oh J, et al. Inflammation, metabolic dysregulation, and pulmonary function among obese urban adolescents with asthma. Am J Respir Crit Care Med 2015; 191: 149-160.

29 Forno E, Weiner DJ, Mullen J, et al. Obesity and airway dysanapsis in children with and without asthma. Am J Respir Crit Care Med 2017; 195: 314-323.

30 Postma DS, Brightling C, Baldi S, et al. Exploring the relevance and extent of small airways dysfunction in asthma (ATLANTIS): baseline data from a prospective cohort study. Lancet Respir Med 2019; 7: 402-416.

31 Watson RA, Pride NB, Thomas EL, et al. Reduction of total lung capacity in obese men: comparison of total intrathoracic and gas volumes. J Appl Physiol 2010; 108: 1605-1612.

32 Kaminsky DA, Irvin CG, Lundblad LK, et al. Heterogeneity of bronchoconstriction does not distinguish mild asthmatic subjects from healthy controls when supine. J Appl Physiol 2008; 104: 10-19.

33 Orfanos S, Jude J, Deeney BT, et al. Obesity increases airway smooth muscle responses to contractile agonists. Am J Physiol Lung Cell Mol Physiol 2018; 315: L673-L681.

34 Nie Z, Jacoby DB, Fryer AD. Hyperinsulinemia potentiates airway responsiveness to parasympathetic nerve stimulation in obese rats. Am J Respir Cell Mol Biol 2014; 51: 251-261.

35 Holguin F, Grasemann H, Sharma S, et al. L-citrulline as add-on therapy to increase nitric oxide, and to improve asthma control in obese asthmatics. JCI Insight 2019; 4: e131733.

36 Wagers S, Lundblad LK, Ekman M, et al. The allergic mouse model of asthma: normal smooth muscle in an abnormal lung? J Appl Physiol 2004; 96: 2019-2027.

37 Periyalil HA, Wood LG, Wright TA, et al. Obese asthmatics are characterized by altered adipose tissue macrophage activation. Clin Exp Allergy 2018; 48: 641-649. 
Halpern D, Grotberg JB. Surfactant effects on fluid-elastic instabilities of liquid-lined flexible tubes: a model of airway closure. J Biomech Eng 1993; 115: 271-277.

39 Seah AS, Grant KA, Aliyeva M, et al. Quantifying the roles of tidal volume and PEEP in the pathogenesis of ventilator-induced lung injury. Ann Biomed Eng 2011; 39: 1505-1516.

40 van Huisstede A, Rudolphus A, Castro Cabezas M, et al. Effect of bariatric surgery on asthma control, lung function and bronchial and systemic inflammation in morbidly obese subjects with asthma. Thorax 2015; 70 : 659-667. 